\title{
Heritability of sow longevity and lifetime prolificacy in Finnish Yorkshire and Landrace pigs
}

\author{
Marja-Liisa Sevón-Aimonen and Pekka Uimari \\ MTT Agrifood Research Finland, Biotechnology and Food Research, Biometrical Genetics, Fl-31600 Jokioinen, Finland \\ e-mail: marja-liisa.sevon-aimonen@mtt.fi
}

\begin{abstract}
The objective of this study was to estimate genetic parameters for longevity traits in Finnish pig populations. Analyzed traits were length of productive herd life (LPL), total number of parities (TNP), total number of piglets born (TNB), total number born alive (TBA) and stillborn (TSB), percentage stillborn (SB\%), total number of piglets dead before weaning (TPM), mortality percentage (PM\%), and total number weaned (TNW). Data contained litter records from 29805 Finnish Landrace and 25807 Finnish Yorkshire sows. Variance components were estimated using the Al-REML method. Heritability estimates varied from 0.06 to 0.11 in Finnish Landrace and from 0.09 to 0.12 in Finnish Yorkshire. Genetic correlations were high (>0.9) between LPL, TNP, TNB, TBA, and TNW, and low between piglet mortality traits (SB\% and PM\%) and other longevity traits. The obtained heritability estimates indicate that there is sufficient genetic variation for selection.
\end{abstract}

Key words: genetic correlation, piglets, selection, variance components

\section{Introduction}

Longevity of sows has a major impact on the efficiency of piglet production by reducing the number of gilts needed for replacement and by increasing the proportion of sows in their highest production phase (Serenius and Stalder 2006, Sasaki and Koketsu 2008, Hoge and Bates 2011). Although culling is eventually a decision made by a farmer culling of sows, especially young sows, is rarely made voluntarily. It has been estimated that in USA a sow must produce at least three litters to be profitable (Stalder et al. 2003). The same holds for Finnish pork production (Niemi et al. 2011). The optimal time to replace a sow depends on factors such as the sow's performance, its housing, feeding, and insemination costs, and the cost and genetic merit of the replacement gilt compared to the sow being considered.

The survival of primiparous sows to the second parity is the single most important phase for their overall longevity. The probability to survive is highest for fat, slow-growing gilts that produce large litters and have good feed intake during lactation as well as enough fat at weaning (Tummaruk et al. 2001, Knauer et al. 2010, Hoge and Bates 2011, Lewis and Bunter 2011). These can be ensured through management practices, e.g. by mating gilts on their third oestrus cycle (Cottney et al. 2012), and by feeding fast-growing and lean pigs with low-protein diets before and during their first pregnancy to promote fat deposition in the body and with high-energy diets during lactation to preserve the fat reserves (O'Dowd et al. 1997).

Sow longevity can be improved directly by favoring animals with high genetic potential for longevity-related traits or for traits that have a favorable genetic correlation with longevity. Commonly used longevity measures include stayability to a certain age, parity or production level, length of productive life (LPL) measured either from age at first insemination or first farrowing, total number of parities produced before culling (TNP), lifetime prolificacy, and pigs produced per day of life (Holder et al. 1995, Yazdi et al. 2000, Serenius and Stalder 2004, Hoge and Bates 2011). Longevity traits like LPL are generally complex and combine several traits related to fertility, nursing and health, e.g. number of parities, number of piglets born alive and weaned, farrowing interval, milk production, and leg health.

The objective of this study was to estimate the heritabilities and genetic correlations of LPL, TNP, and lifetime prolificacy traits in Finnish Landrace and Yorkshire pig populations. 


\section{Material and methods}

Data

The study data were obtained from the pig breeding company Figen Oy, which operates the Finnish litter recording scheme, and contained records from 29805 Finnish Landrace and 25807 Finnish Yorkshire sows born between 2000 and 2006. In addition, the data contained pedigree information of 17999 Landrace and 15523 Yorkshire animals. Only sows with at least one farrowing record and culling day available were included in the analysis. Also, if the culling was based on any systematic reason (e.g. age, low progeny test result), the record was excluded from the analysis. The analyzed traits were length of productive herd life (LPL), total number of parities (TNP), lifetime prolificacy measured as total number of piglets born (TNB), total number of piglets born alive (TBA), total number of stillborn piglets (TSB), percentage of stillborn piglets (SB\%), total number of piglets dead before weaning (TPM), mortality percentage (PM\%), and total number of weaned piglets (TNW). We determined LPL as the number of days from age at first farrowing to age at culling, as in Serenius and Stalder (2004), SB\% as TSB/TNB, and PM\% as TPM/TBA.

\section{Statistical analyses}

Variance components and genetic and phenotypic correlations were estimated using the Al-REML method in the DMU program package (Madsen et al. 2006). The statistical model used in the analysis was:

$y_{i j k l}=\mu+h_{i}+b_{j}+d_{k}+a_{1}+e_{i j k l}$

where $y_{i j k l}$ is the observation; $h_{i}$ is the fixed effect of herd; $b_{j}$ is the fixed effect of the birth month; $d_{k}$ is the random dam effect (mother of sow); $a$, is the random additive genetic effect of the animal (sow whose longevity was measured); and $e_{i j k k^{\prime}}$ is the residual term. The animal, dam, and residual effects were assumed normally distributed $\mathrm{N}(0, \mathrm{~A} \otimes \mathrm{G}), \mathrm{N}(0, \mathrm{I} \otimes \mathrm{L})$, and $\mathrm{N}(0, \mathrm{I} \otimes \mathrm{R})$ with the variance-covariance structure $\mathrm{V}(a)=\mathrm{A} \sigma_{a^{\prime}}{ }^{\prime} \mathrm{V}(c)=\mathrm{I \sigma} 2_{c^{\prime}} \mathrm{V}(e)=\mathrm{I} \sigma_{e^{\prime}}$, and $\operatorname{cov}(a, e)=0$, where I is the identity matrix; $\mathrm{A}$ is the additive genetic relationship matrix between animals; and $\mathrm{G}$, $L$, and $R$ denote the variance-covariance matrices of the studied traits for additive genetic, dam and residual effects, respectively. Heritabilities were estimated by single-trait analysis and correlations by multi-trait analysis. Due to dependencies between traits (e.g. TNB = TBA + TSB), the estimates are from different multi-trait analyses with different combinations of traits, e.g. analysis 1: LPL, TNP, TNB, TBA, and TNW; analysis 2: LPL, TNP, TNB, TSB, and TPM, etc.

\section{Results}

Descriptive statistics of the studied traits are given in Table 1. The available data show that the average Finnish Landrace sow gives birth to 34.6 live piglets during its lifetime, of which 27.4 piglets survive to weaning. This is equal to 1.1 live piglets and 0.2 weaned piglets more than the lifetime production of the typical Finnish Yorkshire sow. However, the differences in the lifetime prolificacy measures between the two breeds are not due to the bigger litter size of Finnish Landrace, but to their longer herd life. Finnish Landrace sows stay in the herd for 482 days compared to 452 days for Finnish Yorkshire, and Landrace also have more litters during their life (3.29 vs. 3.19). On the other hand, pig mortality is slightly lower in purebred Finnish Yorkshire than Landrace litters. On average $14.1 \%$ of piglets born alive during a sow's lifetime will not survive to weaning in purebred Finnish Landrace litters, whereas the percentage in Finnish Yorkshire is 12.7. The same difference is seen in the total number of piglets that die before weaning, which is 0.3 piglets more for Finnish Landrace than Finnish Yorkshire (Table 1).

The reason why TBA does not equal the sum of TNW and TPM in Table 1 is that there were fewer records for TNW and TPM compared to the other traits. The number of parities of sows for which TNW and TPM were available were 3.01 and 2.95 on average compared to 3.29 and 3.19 for the other traits for Finnish Landrace and Yorkshire, respectively (data not shown here). 
Table 1. Number of observations (N), means and SD of length of productive life (LPL), total number of parities (TNP), and lifetime prolificacy traits in Finnish Landrace and Yorkshire.

\begin{tabular}{lrrrrrr}
\hline & \multicolumn{3}{c}{ Landrace } & \multicolumn{3}{c}{ Yorkshire } \\
& $\mathrm{N}$ & Mean & SD & $\mathrm{N}$ & Mean & SD \\
\hline LPL (in days) & 29802 & 481.5 & 391.9 & 25802 & 452.1 & 377.7 \\
TNP & 29805 & 3.3 & 2.4 & 25807 & 3.2 & 2.3 \\
TNB $^{\text {a }}$ & 29805 & 38.4 & 30.1 & 25807 & 37.1 & 29.2 \\
TBA & 29805 & 34.6 & 27.4 & 25807 & 33.5 & 26.4 \\
TSB & 29805 & 3.8 & 4.4 & 25807 & 3.7 & 4.5 \\
SB\% & 29805 & 10.5 & 11.4 & 25807 & 10.3 & 11.6 \\
TPM & 22764 & 4.7 & 5.4 & 19304 & 4.3 & 5.2 \\
PM\% & 22661 & 14.1 & 13.3 & 19167 & 12.7 & 12.7 \\
TNW & 22764 & 27.4 & 22.7 & 19304 & 27.2 & 22.0 \\
\hline
\end{tabular}

a Total number of piglets born (TNB), total number of piglets born alive (TBA), total number of stillborn piglets(TSB), percentage of stillborn piglets (SB\%), total number of piglets dead before weaning (TPM), mortality percentage (PM\%), and total number of piglets weaned (TNW).

\section{Heritabilities}

Heritability estimates for the studied longevity traits including mortality traits varied from 0.06 (TSB) to 0.11 (SB\%) in Finnish Landrace (Table 2). Slightly higher estimates were obtained for Finnish Yorkshire, from 0.09 (TNW) to 0.12 (TSB, SB\%, and TPM) (Table 3). All estimates had low standard errors (from 0.01 to 0.02 ). The largest difference in heritability estimates between the two breeds was for TSB: $0.06( \pm 0.01)$ in Finnish Landrace and $0.12( \pm$ 0.01 ) in Finnish Yorkshire. There is no obvious explanation for the observed difference.

Table 2. Heritabilities $\left(h^{2} \pm S E\right)$, relative variance due to permanent environmental effect of the dam ( $\left.c^{2} \pm S E\right)$, and phenotypic variances $\left(\mathrm{s}^{2}\right)$ of length of productive life (LPL), total number of parities (TNP), and lifetime prolificacy traits in Finnish Landrace and Yorkshire.

\begin{tabular}{lrrrrrr} 
& \multicolumn{2}{c}{ Landrace } & & & \multicolumn{2}{c}{ Yorkshire } \\
& $\mathrm{h}^{2}$ & $\mathrm{c}^{2}$ & $\mathrm{~s}^{2}$ & $\mathrm{~h}^{2}$ & $\mathrm{c}^{2}$ & \\
\hline LPL (in days) & $0.08 \pm 0.01$ & $0.06 \pm 0.01$ & 138816 & $0.10 \pm 0.01$ & $0.06 \pm 0.01$ & 127361 \\
TNP & $0.08 \pm 0.01$ & $0.06 \pm 0.01$ & 5.1 & $0.10 \pm 0.01$ & $0.06 \pm 0.01$ & 4.6 \\
TNB & $0.08 \pm 0.01$ & $0.05 \pm 0.01$ & 836.6 & $0.11 \pm 0.01$ & $0.05 \pm 0.01$ & 774.9 \\
TBA & $0.09 \pm 0.01$ & $0.05 \pm 0.01$ & 691.8 & $0.11 \pm 0.01$ & $0.05 \pm 0.01$ & 634.5 \\
TSB & $0.06 \pm 0.01$ & $0.04 \pm 0.01$ & 17.9 & $0.12 \pm 0.01$ & $0.03 \pm 0.01$ & 18.1 \\
SB\% & $0.11 \pm 0.01$ & $0.02 \pm 0.01$ & 126.2 & $0.12 \pm 0.01$ & $0.02 \pm 0.01$ & 127.8 \\
TPM & $0.10 \pm 0.01$ & $0.04 \pm 0.01$ & 26.5 & $0.12 \pm 0.02$ & $0.04 \pm 0.01$ & 24.5 \\
PM\% & $0.08 \pm 0.01$ & $0.03 \pm 0.01$ & 161.4 & $0.11 \pm 0.02$ & $0.04 \pm 0.01$ & 151.2 \\
TNW & $0.09 \pm 0.01$ & $0.05 \pm 0.01$ & 462.1 & $0.09 \pm 0.01$ & $0.05 \pm 0.01$ & 432.8 \\
\hline
\end{tabular}

a Total number of piglets born (TNB), total number of piglets born alive (TBA), total number of stillborn piglets(TSB), percentage of stillborn piglets (SB\%), total number of piglets dead before weaning (TPM), mortality percentage (PM\%), and total number of piglets weaned (TNW).

\section{Permanent environmental effect}

Relative variances due to permanent environmental effect of dam (c2) varied for the studied traits from 0.02 (SB\%) to 0.06 (LPL, TNP) for both breeds (Table 2). The standard errors were low (0.01) for all traits. No differences in $\mathrm{c}^{2}$ between the breeds were observed. 


\section{Genetic and phenotypic correlations}

The genetic (0.94 to 0.99) and phenotypic correlations (0.94 to 0.99) between LPL, TNP, TNB, TBA, and TNW were very strong in both breeds (Tables 3 and 4). Sows that stay longer in production will give birth to more piglets during their herd life, and vice versa, sows that produce large litters in every parity will stay in the herd longer than sows producing small litters. Overall, the genetic correlations between the longevity traits were slightly higher in Finnish Landrace than in Finnish Yorkshire (Tables 3 and 4).

Table 3. Genetic ( $\pm \mathrm{SE}$; above the diagonal), and phenotypic (below the diagonal) correlations of length of productive life (LPL, days), total number of parities (TNP), and lifetime prolificacy traits in Finnish Landrace.

\begin{tabular}{llllcccccc}
\hline & LPL & \multicolumn{1}{c}{ TNP } & \multicolumn{1}{c}{ TNB $^{\text {a }}$} & TBA & TSB & SB\% & TPM & PM\% & TNW \\
\hline LPL & & $0.98 \pm 0.01$ & $0.94 \pm 0.01$ & $0.94 \pm 0.01$ & $0.44 \pm 0.07$ & $-0.39 \pm 0.08$ & $0.60 \pm 0.06$ & $-0.05 \pm 0.11$ & $0.96 \pm 0.01$ \\
TNP & 0.96 & & $0.95 \pm 0.01$ & $0.95 \pm 0.01$ & $0.45 \pm 0.07$ & $-0.38 \pm 0.08$ & $0.64 \pm 0.05$ & $-0.01 \pm 0.10$ & $0.97 \pm 0.01$ \\
TNB & 0.93 & 0.97 & & $0.99 \pm 0.00$ & $0.55 \pm 0.06$ & $-0.37 \pm 0.08$ & $0.79 \pm 0.04$ & $0.15 \pm 0.09$ & $0.98 \pm 0.00$ \\
TBA & 0.93 & 0.96 & 0.99 & & $0.45 \pm 0.07$ & $-0.44 \pm 0.07$ & $0.41 \pm 0.08$ & $0.10 \pm 0.11$ & $0.99 \pm 0.00$ \\
TSB & 0.59 & 0.62 & 0.66 & 0.57 & & $0.58 \pm 0.06$ & $0.38 \pm 0.07$ & $0.03 \pm 0.12$ & $0.46 \pm 0.07$ \\
SB\% & -0.10 & -0.08 & -0.07 & -0.15 & 0.48 & & $-0.40 \pm 0.08$ & $-0.24 \pm 0.06$ & $-0.44 \pm 0.07$ \\
TPM & 0.62 & 0.64 & 0.73 & 0.43 & 0.47 & -0.09 & & $0.72 \pm 0.05$ & $0.71 \pm 0.05$ \\
PM\% & -0.03 & -0.02 & 0.04 & 0.04 & 0.04 & 0.00 & 0.50 & & $-0.00 \pm 0.10$ \\
TNW & 0.94 & 0.97 & 0.98 & 0.99 & 0.61 & -0.14 & 0.63 & -0.11 & \\
\hline
\end{tabular}

${ }^{a}$ Total number of piglets born (TNB), total number of piglets born alive (TBA), total number of stillborn piglets (TSB), percentage of stillborn piglets (SB\%), total number of piglets dead before weaning (TPM), mortality percentage (PM\%), and total number of piglets weaned (TNW).

Table 4. Genetic ( \pm SE; above the diagonal), and phenotypic (below the diagonal) correlations of length of productive life (LPL, days), total number of parities (TNP), and lifetime prolificacy traits in Finnish Yorkshire.

\begin{tabular}{llllllllll}
\hline & LPL & TNP & TNB $^{a}$ & TBA & TSB & SB\% & TPM & PM\% & TNW \\
\hline LPL & & $0.97 \pm 0.01$ & $0.94 \pm 0.01$ & $0.94 \pm 0.01$ & $0.51 \pm 0.06$ & $-0.10 \pm 0.09$ & $0.58 \pm 0.06$ & $-0.15 \pm 0.10$ & $0.94 \pm 0.01$ \\
TNP & 0.97 & & $0.95 \pm 0.06$ & $0.95 \pm 0.01$ & $0.53 \pm 0.06$ & $-0.08 \pm 0.09$ & $0.56 \pm 0.06$ & $-0.18 \pm 0.10$ & $0.96 \pm 0.01$ \\
TNB & 0.94 & 0.97 & & $0.99 \pm 0.01$ & $0.60 \pm 0.05$ & $-0.07 \pm 0.09$ & $0.69 \pm 0.05$ & $-0.06 \pm 0.10$ & $0.97 \pm 0.00$ \\
TBA & 0.94 & 0.96 & 0.99 & & $0.50 \pm 0.06$ & $-0.20 \pm 0.08$ & $0.39 \pm 0.07$ & $0.00 \pm 0.10$ & $0.98 \pm 0.00$ \\
TSB & 0.56 & 0.59 & 0.66 & 0.57 & & $0.74 \pm 0.05$ & $0.52 \pm 0.07$ & $0.21 \pm 0.09$ & $0.56 \pm 0.06$ \\
SB\% & -0.07 & -0.05 & -0.04 & -0.13 & 0.49 & & $-0.06 \pm 0.09$ & $0.19 \pm 0.10$ & $-0.20 \pm 0.06$ \\
TPM & 0.59 & 0.61 & 0.71 & 0.43 & 0.50 & -0.06 & & $0.70 \pm 0.06$ & $0.56 \pm 0.07$ \\
PM\% & 0.02 & 0.04 & 0.09 & 0.09 & 0.09 & 0.00 & 0.55 & & $-0.25 \pm 0.09$ \\
TNW & 0.94 & 0.96 & 0.98 & 0.99 & 0.60 & -0.12 & 0.61 & 0.05 & \\
\hline
\end{tabular}

${ }^{a}$ Total number of piglets born (TNB), total number of piglets born alive (TBA), total number of stillborn piglets (TSB), percentage of stillborn piglets (SB\%), total number of piglets dead before weaning (TPM), mortality percentage (PM\%), and total number of piglets weaned (TNW).

Moderate genetic correlations were obtained between TSB and lifetime prolificacy traits in both breeds, from 0.44 to 0.55 in Finnish Landrace (Table 3) and from 0.50 to 0.60 in Finnish Yorkshire (Table 4). The genetic correlations for TPM and lifetime prolificacy traits were slightly higher, varying from 0.41 to 0.79 (Table 3 ) in Finnish Landrace and from 0.39 to 0.69 (Table 4) in Finnish Yorkshire. Low correlations were found between LPL, TNP, lifetime prolificacy traits, and piglet mortality when mortality was measured as SB\% or PM\%. The explanation for the differences in genetic correlations between mortality measured as a proportion and as an absolute number is that sows with a longer productive life will also have a higher absolute number but not necessarily a higher proportion of dead piglets. Genetic correlations of SB\% also showed a notable difference between breeds. The estimates were three to four times higher in Finnish Landrace than in Finnish Yorkshire. A detailed investigation of the variance-covariance components for SB\% revealed that although the sum of the covariances for the dam and animal effects were 
similar in both breeds, the model and the method were unable to distinguish between the effects of the dam and the animal for this particular trait. The reason may lie in the structure of the available data where the proportion of dams with only one daughter was relatively high (approx. $37 \%$ in both breeds) compared to dams with multiple daughters. All genetic covariance estimates related to the mortality traits had large standard errors (from 0.04 to 0.12). Based on the observed genetic covariances, selection for longevity traits will not increase piglet mortality.

\section{Discussion}

The culling of a sow is a decision made by the farmer. Therefore, it does not exactly describe the true longevity of sows. In order to avoid biases in heritability estimates sows which were clearly culled based on voluntary culling reasons, such as age of the sow, were excluded from analysis. Previously reported heritability estimates for LPL range from 0.10 to 0.22 in Finnish Yorkshire and from 0.05 to 0.17 for Finnish Landrace, using either a linear or a survival model, respectively (Serenius and Stalder 2004, Serenius et al. 2008). Thus, the estimates reported in earlier studies have also been higher for Finnish Yorkshire than Finnish Landrace. The estimates presented in our study correspond well with those obtained previously from the linear model (Serenius and Stalder, 2004). In other breeds the heritability estimates for LPL have ranged from very low (0.02) to moderate (0.31), depending on the population and the analysis method (Tholen et al. 1996, López-Serrano et al. 2000, Guo et al. 2001, Engblom et al. 2009, Mészáros et al. 2010).

Serenius and Stalder (2004) also reported a very high (0.96) positive genetic correlation between LPL and TBA. In the same study, the genetic correlation between LPL and the number of weaned piglets in the first litter was moderate (0.39), implying that the results from the first litter give a positive but not very strong indication of a sow's genetic potential to stay long in production. Further, no genetic correlation was found between LPL or TBA and backfat thickness at $100 \mathrm{~kg}$ of live weight, which means that backfat thickness cannot be used as an indicator trait of sow longevity. Better progress in longevity can be achieved by selecting sows with a high leg conformation score (Serenius and Stalder, 2004) or a short weaning-to-conception interval after the first farrowing (Tholen et al. 1996).

The heritability estimates obtained for sow longevity traits in Finnish Yorkshire and Finnish Landrace were similar to those for the reproductive traits, indicating that there is sufficient genetic variation for selection in both breeds. The reproductive traits of sows are currently estimated in the Finnish national evaluation system by multi-trait analysis, which can be expanded to include longevity traits as well. Given the high genetic correlations between LPL, TNP, TNB, TBA, and TNW in both breeds it would be reasonable to include only one of these traits into the multi-trait analysis and the total merit index, in order to avoid an unnecessarily heavy computational burden. The most suitable trait would be TNP, because it is very easy to measure, does not require an exact culling date, and is free of possible errors in litter size records due to occasional involuntary or voluntary mixing of piglets between sows that farrowed at the same time. Further, because the observed genetic correlations between piglet mortality (SB\% and PM\%) and TNP were low (except for between SB\% and TNP in Finnish Landrace), selection for TNP is not expected to increase piglet mortality. Given that longevity can only be measured after culling, the most promising approach for genetic improvement of longevity is through genomic selection. Finnish pig breeds are, in fact, particularly suitable for genomic selection because of the uniformity of the breeds (Uimari and Tapio 2011). Currently all boars entering Al in Finland are genotyped with a high-density SNP panel (PorcineSNP60 BeadChip, Illumina Ltd., San Diego, USA). The estimates of the variance components given in this article allow estimation of the breeding values of the reference boars and creation a prediction equation for genomic selection. Alternatively, the pedigree, phenotypic, and genomic information can be combined into a single-step procedure (Misztal et al. 2009). Additionally, data from the same population s will be analyzed using a survival model that allows the use of the censored records typical for the large proportion of recent sows in the population.

\section{Acknowledgements}

Figen Oy is gratefully acknowledged for providing the phenotypic and pedigree data for this study. This work was supported by the Finnish Ministry of Agriculture and Forestry. 


\section{References}

Cottney, P.D., Magowan, E., Ball, M.E.E. \& Gordon, A. 2012. Effect of oestrus number of nulliparous sows at first service on first litter and lifetime performance. Livestock Science 146: 5-12.

Engblom, L., Lundeheim, N., Schneider, M.P., Dalin, A.M. \& Andersson, K. 2009. Genetics of crossbred sow longevity. Animal 3: 783-790.

Guo, S.F., Gianola, D., Rekaya, R. \& Short, T. 2001. Bayesian analysis of lifetime performance and prolificacy in Landrace sows using a linear mixed model with censoring. Livestock Production Science 72: 243-252.

Hoge, M.D. \& Bates, R.O. 2011. Developmental factors that influence sow longevity. Journal of Animal Science 89: 1238-1245.

Holder, R.B., Lamberson, W.R., Bates, R.O. \& Safranski, T.J. 1995. Lifetime productivity in gilts previously selected for decreased age at puberty. Animal Science 61: 115-121.

Knauer. M., Stalder, K.J., Serenius, T., Baas, T.J., Berger, P.J., Karriker, L., Goodwin, R.N., Johnson, R.K., Mabry, J.W., Miller, R.K., Robison, O.W. \& Tokach, M.D. 2010. Factors associated with sow stayability in 6 genotypes. Journal of Animal Science 88: 3486-3492.

Lewis, C.R.G. \& Bunter, K.L. 2011. Body development in sows, feed intake and maternal capacity. Part 2: gilt body condition before and after lactation, reproductive performance and correlations with lactation feed intake. Animal 5: 1855-1867.

López-Serrano, M., Reinsch, N., Looft, H. \& Kalm, E. 2000. Genetic correlations of growth, backfat thickness and exterior with stayability in large white and landrace sows. Livestock Production Science 64: 121-131.

Madsen, P., Sørensen, P., Su, G., Damgaard, L.H., Thomsen, H. \& Labouriau, R. 2006. DMU - A package for analyzing multivariate mixed models. In: German Society for animal Science (ed.). Proceedings of the World Congress on Genetics Applied to Livestock Production. Belo Horizonte, Brazil. CD communication: 27-11.

Mészáros, G., Pálos, J., Ducrocq, V. \& Sölkner, J. 2010. Heritability of longevity in Large White and Landrace sows using continuous time and grouped data models. Genetics Selection Evolution 42: 13.

Misztal, I., Legarra, A. \& Aguilar, I. 2009. Computing procedures for genetic evaluation including phenotypic, full pedigree, and genomic information. Journal of Dairy Science 92: 4648-4655.

Niemi, J., Sevón-Aimonen, M.-L., Partanen K. \& Pietola, K. 2011. Factors contributing to sow replacement and the value of prolonged productive life. In: The 24th NJF Congress: Food, Feed, Fuel and Fun, Book of abstracts. NJF Report 7 3: p. 59.

O'Dowd, S., Hoste, S., Mercer, J.T., Fowler, V.R. \& Edwards, S.A. 1997. Nutritional modification of body composition and the consequences for reproductive performance and longevity in genetically lean sows. Livestock Production Science 52: 155-165.

Sasaki, Y. \& Koketsu, Y. 2008. Sows having high lifetime efficiency and high longevity associated with herd productivity in commercial herds. Livestock Science 118: 140-146.

Serenius, T. \& Stalder, K.J. 2004. Genetics of length of productive life and lifetime prolificacy in the Finnish Landrace and Large White pig populations. Journal of Animal Science 82: 3111-3117.

Serenius, T. \& Stalder, K.J. 2006. Selection for sow longevity. Journal of Animal Science 84: E166-E171.

Serenius, T., Stalder, K.J. \& Fernando, R.L. 2008. Genetic associations of sow longevity with age at first farrowing, number of piglets weaned, and wean to insemination interval in the Finnish Landrace swine population. Journal of Animal Science 86: 3324-3329.

Stalder, K.J., Lacy, R.C., Cross, T.L. \& Conatser, G.E. 2003. Financial impact of average parity of culled females in a breed-to-wean swine operation using replacement gilt net present value analysis. Journal of Swine Health and Production 11: 69-74.

Tholen, E., Bunter, K.L., Hermesch, S. \& Graser, H.U. 1996. The genetic foundation of fitness and reproduction traits in Australian pig populations. 1. Genetic parameters for weaning to conception interval, farrowing interval, and stayability. Australian Journal of Agricultural Research 47: 1261-1274.

Tummaruk, P., Lundeheim, N., Einarsson, S. \& Dalin, A.M. 2001. Effect of birth litter size, birth parity number, growth rate, backfat thickness and age at first mating of gilts on their reproductive performance as sows. Animal Reproduction Science 66: 225-237.

Uimari, P. \& Tapio, M. 2011. Extent of linkage disequilibrium and effective population size in Finnish Landrace and Finnish Yorkshire pig breeds. Journal of Animal Science 89: 609-614.

Yazdi, M., Rydhmer, L., Ringmar-Cederberg, E., Lundeheim, N. \& Johansson, K. 2000. Genetic study of longevity in Swedish Landrace sows. Livestock Production Science 63: 255-264. 